\title{
BMJ Open Multicentre endoscopist-blinded randomised clinical trial to compare two bowel preparations after a colonoscopy with inadequate cleansing: a study protocol
}

\author{
Michael Sai Lai Sey, ${ }^{01,2}$ Daniel von Renteln, ${ }^{3}$ Richard Sultanian, ${ }^{4}$ \\ Cassandra McDonald, ${ }^{2}$ Myriam Martel, ${ }^{5}$ Alan Barkun ${ }^{5}$
}

To cite: Sey MSL, von Renteln D, Sultanian R, et al. Multicentre endoscopistblinded randomised clinical trial to compare two bowel preparations after a colonoscopy with inadequate cleansing: a study protocol. BMJ Open 2019;9:e029573. doi:10.1136/ bmjopen-2019-029573

- Prepublication history and additional material for this paper are available online. To view these files, please visit the journal online (http://dx.doi org/10.1136/bmjopen-2019029573).

Received 31 January 2019 Revised 18 April 2019 Accepted 11 June 2019

Check for updates

(C) Author(s) (or their employer(s)) 2019. Re-use permitted under CC BY-NC. No commercial re-use. See rights and permissions. Published by BMJ.

For numbered affiliations see end of article.

Correspondence to Dr Michael Sai Lai Sey; msey2@uwo.ca

\section{ABSTRACT}

Introduction Inadequate bowel preparation is common and negatively impacts colonoscopy quality. The objective of this study is to compare two bowel preparation regimens in cleansing the colon after an index colonoscopy with failed bowel preparation.

Methods and analysis This is a phase III, multicentre, randomised clinical trial comparing two bowel preparation regimens after failure to adequately cleanse at the index colonoscopy. Regimen A consists of $4 \mathrm{~L}$ split-dose polyethylene glycol electrolyte solution (PEG-ELS) and Regimen B consists of $6 \mathrm{~L}$ split-dose PEG-ELS, both preceded by $15 \mathrm{mg}$ of bisacodyl the day before the procedure along with a low-fibre diet 3 and 2 days before the procedure followed by a clear fluid diet starting the day before the procedure. The primary outcome is adequate bowel preparation, defined as a Boston Bowel Preparation Scale (BBPS) score of $\geq 6$ with each segment score $\geq 2$. Secondary outcomes include mean BBPS score, bowel preparation adequacy using the US Multi-Society Task Force on Colorectal Cancer definition, detection rate by polyp subtype, caecal intubation rate, mean Validated Patient Tolerability Questionnaire for Bowel Preparation score, subject willingness to repeat the preparation and faecal incontinence rate.

Ethics and dissemination The study will be conducted in accordance with Good Clinical Practice guidelines and local institutional standards. Study findings will be disseminated at an international gastroenterology conference and published in peer-reviewed journals. Trial registration number NCT02976805; Pre-results.

\section{INTRODUCTION}

Adequate bowel preparation is a prerequisite for quality colonoscopy. ${ }^{1-3}$ Inadequate bowel cleansing is associated with incomplete procedures and lower polyp and adenoma detection rates. ${ }^{4-7}$ Of greater concern, studies examining findings on follow-up colonoscopies after inadequate bowel preparation have reported adenoma miss rates upwards of $47 \% .^{8-10}$ Furthermore, poor bowel
Strengths and limitations of this study

- First clinical trial to use a supratherapeutic dose of polyethylene glycol electrolyte solution to cleanse the colon after failed bowel preparation for colonoscopy.

- Second clinical trial to investigate how to achieve adequate bowel preparation after a prior failed attempt.

- Rigorous methodology, including multicentre enrollment, concealed randomisation, blinding of endoscopists/outcome assessors and minimisation of study interventions beyond routine clinical practice.

Limitation is inability to blind subjects.

preparation is a common problem and estimated to affect $4 \%-17 \%$ of colonoscopies depending on case definition. ${ }^{411-16}$ Despite this, there is a relative paucity of research in this population. Although there have been many studies investigating bowel preparation in general, relatively few address how to adequately cleanse patients who have previously failed bowel preparation.

Studies that have reported on this topic suffer from significant methodological limitations. In a small case series, Ibanez et $\mathrm{l}^{17}$ gave 51 patients with inadequate bowel preparation an 'intensive' regimen consisting of a 72 hours low-fibre diet, $10 \mathrm{mg}$ of bisacodyl and $3 \mathrm{~L}$ split-dose polyethylene glycol electrolyte solution (PEG-ELS). Using this regimen, 90\% had adequate bowel preparation at the second colonoscopy. Unfortunately, most patients did not receive split-dose bowel preparation at the index colonoscopy, which is now a standard practice due to superior cleansing, and the study did not include a control group. ${ }^{18}$ In another study, 85 patients with inadequate bowel preparation after split-dose PEG-ELS (4L) were offered 
a repeat colonoscopy the same day after ingestion of an additional 2L of PEG-ELS or a colonoscopy 1 week later using a 7-day low-fibre diet, $20 \mathrm{mg}$ of bisacodyl and 4L split-dose PEG-ELS. ${ }^{19}$ There was no difference in adequacy of bowel preparation although the intervention was not randomised, and $20 \%$ of cases were still inadequate. In the only randomised controlled trial published to date, Gimeno-Garcia et al recently randomised 256 patients to $4 \mathrm{~L}$ split-dose PEG-ELS versus $2 \mathrm{~L}$ split-dose PEG-ELS +ascorbicacid, both with a 72 hours low-fibre diet and $10 \mathrm{mg}$ of bisacodyl. ${ }^{20}$ The $4 \mathrm{~L}$ split-dose PEG-ELS regimen was superior although almost $20 \%$ of patients still had inadequate bowel preparation.

To date, there is no widely accepted bowel preparation regimen after failure to adequately cleanse the colon at index colonoscopy. A highly efficacious yet tolerable bowel preparation regimen is needed for these patients to ensure adequate cleansing for their next procedure. The objective of this multi-centre randomised clinical trial is to compare the efficacy of $4 \mathrm{~L}$ split-dose PEG-ELS versus 6L split-dose PEG-ELS, both with $15 \mathrm{mg}$ of bisacodyl and a low fibre diet followed by a clear fluid diet, in achieving adequate bowel preparation after failing to cleanse at the index colonoscopy.

\section{METHODS AND ANALYSIS}

\section{Study design and patient population}

This phase III multicentre randomised clinical trial will compare two bowel preparation regimens for patients who have previously had an inadequate bowel preparation at an index colonoscopy. The study will be conducted at four Canadian centres (Western University, University of Montreal, University of Alberta and McGill University) and involve a total of seven hospitals. The study group was selected from a national consortium of investigators participating in a large multicentre clinical trial on bowel preparation in the general population, The Bowel Cleansing: a National Initiative (B-CLEAN) (ClinicalTrials. gov NCT02547571). As such, we propose naming our trial, The Bowel Cleansing: a National Initiative-Repeat Colonoscopy (B-CLEAN(R), pronounced 'Be Cleaner').

Anyone requiring a repeat outpatient colonoscopy due to a failed bowel preparation at the index colonoscopy is eligible to participate. Failure is defined as preparation quality inadequate to detect lesions $>5 \mathrm{~mm}$ after washing and requiring a shortened time interval to the next procedure as a result. This pragmatic clinical definition, endorsed by the US Multi-Society Task Force (USMSTF) on Colorectal Cancer, was chosen over formal bowel preparation scales for two reasons. ${ }^{21}$ First, it increases generalisability of the study results since it addresses the fundamental goal of bowel preparation, the adequate detection of polyps with sufficient confidence that there is no need to shorten the interval to the next colonoscopy, without committing to a single bowel preparation scale, of which there are many. ${ }^{22}$ Second, it aids in subject recruitment since patients who have their index colonoscopies by non-study physicians would still be eligible to participate regardless of which bowel preparation score was used to determine failure. This definition of inadequate bowel preparation has been widely available since 2002, was endorsed by two USMSTF guidelines, ${ }^{3}{ }^{21}$ used in numerous clinical reviews and societal guidelines on bowel preparation and colonoscopy quality, ${ }^{12324}$ and was in part used to define inadequate bowel preparation for two well-validated bowel preparation scales: the Boston Bowel Preparation Scale (BBPS) and Ottawa Bowel Preparation Quality Scale. ${ }^{22}{ }^{25} 26$ Exclusion criteria for the study include (1) an index colonoscopy where the subject was non-compliant with bowel preparation instruction, used an off-label bowel preparation or had the procedure as an inpatient; (2) is at an increased risk for electrolyte or fluid disturbance with high-volume bowel preparation, such as congestive heart failure, chronic renal failure, cirrhosis or severe electrolyte disturbance; (3) has a general contraindication to bowel preparation or colonoscopy, such as pregnancy or breast feeding, allergies to components of bowel preparation, history of ischaemic colitis (ie, a contraindication for bisacodyl), ileus, gastric outlet obstruction, gastrointestinal obstruction, bowel perforation, toxic colitis, toxic megacolon, acute surgical abdomen including appendicitis, gastroenteritis and acute diverticulitis; (4) age $<18$ years; (5) history of any colonic surgery; (6) inability to follow verbal and written instructions in English or French; (7) lack of an indication for full colonoscopy and (8) subject refusal or inability to comprehend the trial.

\section{Selection of bowel preparation regimens}

All subjects will be given the following dietary instructions (online supplementary appendix A): (1) follow a low-fibre diet 3 and 2 days before the procedure, (2) follow a clear fluid diet the day before the procedure and (3) continue the clear fluid diet on the day of the procedure until 2 hours before the procedure, when fasting begins. In regimen A, subjects will take (1) $15 \mathrm{mg}$ of bisacodyl at 2 PM the day before the procedure, (2) drink $2 \mathrm{~L}$ PEG-ELS at a rate of $240 \mathrm{~mL}$ every $10 \mathrm{~min}$ the night before the procedure starting at $8 \mathrm{PM}$ and (3) drink 2L PEG-ELS at a rate of $240 \mathrm{~mL}$ every $10 \mathrm{~min}$ on the day of the procedure to be started 4-6 hours before the appointment and finished at least 2 hours before the procedure. In regimen $\mathrm{B}$, subjects will take (1) $15 \mathrm{mg}$ of bisacodyl at $2 \mathrm{PM}$ the day before the procedure, (2) drink 4L PEG-ELS at a rate of $240 \mathrm{~mL}$ every $10 \mathrm{~min}$ the night before the procedure starting at 6 PM and (3) drink 2L PEG-ELS at a rate of $240 \mathrm{~mL}$ every $10 \mathrm{~min}$ on the day of the procedure to be started 4-6 hours before the appointment and finished at least 2 hours before the procedure (online supplementary appendix B).

Regimen A was adapted from Ibanez et $a l^{17}$ who used a regimen consisting of 3L PEG-ELS and $10 \mathrm{mg}$ bisacodyl with reasonable effectiveness. In our study, 4L PEG-ELS is used instead of $3 \mathrm{~L}$ to avoid reducing the volume of PEG-ELS consumed by those who were originally prepped 
with 4L PEG-ELS at their index colonoscopy (PegLyte, GoLytely, CoLyte, Pendopharm, Montreal, Canada). Fifteen milligrams of bisacodyl was used instead of $10 \mathrm{mg}$ to avoid a dose reduction since low-volume PEG-ELS with bisacodyl is given as $15 \mathrm{mg}$ in Canada (BiPegLyte, Pendopharm, Montreal, Canada). Regimen B was adapted from Kim $e t a l^{19}$ as a more intensive yet tolerable regimen by adding an additional 2 L PEG-ELS to Regimen A.

\section{Randomisation and blinding}

Subjects will be randomised in a 1:1 allocation in blocks of two to four stratified by site to either Regimen A or B. Randomisation will be performed centrally online using Research Electronic Data Capture (REDCap). REDCap is administered through Lawson Health Research Institute, an affiliate of London Health Sciences Centre, complies with relevant research and hospital privacy guidelines, and all data are stored locally in London, Canada. The randomisation list will be concealed and stored on Western University's REDCap servers with randomisation allocation visible only after passing the study eligibility screening webpage and available one subject at a time. In addition, all study data will be entered securely online through the encrypted REDCap platform.

Blinding of the endoscopist will be strictly enforced. Endoscopists are required to remain blinded to the subject's bowel preparation until after completing the study case report form. As such, study enrollment and randomisation will be performed by research staff. Subjects will be asked to refrain from discussing the bowel preparation with clinical staff until after the colonoscopy. Unfortunately, blinding of the patients is not possible due to the volume differences between the two bowel preparation regimens.

\section{Colonoscopy procedures}

All colonoscopies will be performed within 12 weeks of randomisation but not within 2 weeks of the index colonoscopy, which will serve as a washout period from the index bowel preparation. Subjects will be taught how to take their bowel preparation and be given written instructions for diet along with dose and timing of bowel preparation medications according to randomisation. Bisacodyl and PEG-ELS will be provided free-of-charge, but there is no other study remuneration or compensation. The only study-specific contact will be a phone call 14 days after the procedure to assess for adverse events. Otherwise, there are no study-specific reminders, phone calls or encounters. Colonoscopies will be performed according to local standard operating procedures. All participating endoscopists will complete standardised training in the use of the BBPS prior to the start of the study(at http://www. bmc.org/gastroenterology/research.htm). A record of training will be sent to the coordinating centre.

For subjects who do not present for their colonoscopy after randomisation, such as those who forget to attend their appointments, they may remain in the study and be prepped with the same regimen. For subjects who decline ongoing study participation, they can withdraw at any time and follow up with their physician.

\section{Baseline data collection}

The following variables will be collected at baseline: age; sex; weight; height; primary language; highest level of education; patient's ability to understand and follow the bowel preparation instructions as deemed by the research personnel; Charleston comorbidity index score $;^{27}$ history of constipation predominate irritable bowel syndrome defined by the ROME III criteria $;^{28}$ history of functional constipation defined by the ROME III criteria; ${ }^{28}$ history of neurologic disorders, such as Parkinson's disease, multiple sclerosis, cerebral palsy; previous abdominal/ pelvic surgery; established diagnosis of inflammatory bowel disease; usage of narcotics at least once/week; laxatives at least once/week; calcium channel blocker on a daily basis; information regarding index colonoscopy, such as method of communication for index colonoscopy, bowel preparation used, whether it was given in a split-dose manner, segmental and total BBPS score when available, patient compliance, willingness to repeat index colonoscopy bowel preparation, subject incontinence and travel time with index bowel preparation and indication for index colonoscopy.

\section{Study outcomes}

The primary outcome will be adequate bowel preparation, defined as a BBPS total score $\geq 6$ and/or individual segment scores $\geq 2 .{ }^{25} 2629$ The BBPS was selected for use since it is the most thoroughly validated bowel preparation scale based on a recent systematic review, has substantial to excellent inter-observer reliability (ICC $=0.74-0.91$ ) and is already widely used today. ${ }^{22}$ The total score ranges between 0 and 9 and is based on three colonic segment scores (right, transverse and left), each rated between 0 and 3 as follows: $3=$ entire mucosa of colon segment seen well with no residual staining, small fragments of stool or opaque liquid; $2=$ minor amount of residual staining, small fragments of stool and/or opaque liquid, but mucosa of colon segment seen well; 1 =portion of mucosa of the colon segment seen, but other areas of the colon segment not well seen because of staining, residual stool and/or opaque liquid; $0=$ unprepared colon segment with mucosa not seen because of solid stool that cannot be cleared. Secondary outcomes include mean BBPS total score, right-sided BBPS sub-score, bowel preparation adequacy defined as the ability to detect lesions $>5 \mathrm{~mm}$ in size after washing without a need to shorten the interval to the next colonoscopy, detection rate by histology subtype (polyp, adenoma, advanced adenoma $(>1 \mathrm{~cm}$, villous component, sessile serrated polyp/adenoma, or high-grade dysplasia), adenocarcinoma, polyp per colonoscopy and adenoma per colonoscopy), caecal intubation rate, mean Validated Patient Tolerability Questionnaire for Bowel Preparation score (online supplementary appendix C), ${ }^{30}$ subject product completion ( $\%$ of total required intake), subject willingness to repeat the preparation and faecal 
incontinence rate. Patient dietary compliance will be assessed with a food diary for the 3 days before colonoscopy and compliance with bowel preparation medications assessed with a take home form to be completed by the patient as they consume their medications.

\section{Adverse events}

There are no anticipated adverse events related to participation in this study beyond that inherent to bowel preparation for colonoscopy in general. Although the study uses a higher volume of PEG-ELS, we do not anticipate any serious adverse events (SAEs) due to the safety profile of PEG-ELS, which is electrolyte and fluid balanced. Furthermore, high volumes of PEG-ELS given rapidly are already used in clinical practice. As an example, $1 \mathrm{~L}$ of PEG-ELS given every 30-45 min until the effluent is clear (usually requiring at least $4 \mathrm{~L}$ over 2 hours) can be given in the setting of acute lower gastrointestinal bleeding. ${ }^{31}$ Similarly, $4 \mathrm{~L}$ of PEG-ELS can be given over 4 hours to rapidly treat hepatic encephalopathy. ${ }^{32}$ Lastly, $6 \mathrm{~L}$ of PEG-ELS has already been used in a study of 85 patients with failed bowel prep without untoward effects. ${ }^{19}$ Thus, we expect the use of $6 \mathrm{~L}$ split-dose PEG-ELS in regimen $\mathrm{B}$ of the study to be safe. In addition, patients at risk for fluid and electrolyte disturbances will be excluded. Nonetheless, adverse events will be assessed at the time of colonoscopy and by a phone call 14 days after the procedure. All adverse events related to either bowel preparation or colonoscopy will be recorded and communicated to the data coordinating centre (Western University), the local REB and Health Canada as required.

The following moderate and non-lasting symptoms related to bowel preparation are expected and are not to be considered an adverse event: nausea, vomiting, abdominal fullness, bloating, abdominal cramps and pain, diarrhoea and anal irritation. Instead, these known symptoms of bowel preparation will be captured in the Validated Patient Tolerability Questionnaire for Bowel Preparation form. An SAE is defined as an event that led to death, led to fetal distress, fetal death or a congenital abnormality or birth defect, or led to a serious deterioration in the health of the subject that resulted in a life-threatening illness or injury, resulted in permanent impairment of a body structure or a body function, required in-subject hospitalisation or prolongation of existing hospitalisation, resulted in medical or surgical intervention to prevent permanent impairment to a body structure or a body function. Causality terms, as related to the study drug and procedure, are defined as follows: unrelated if the adverse event is determined to be due to a concurrent illness or effect of another (device) drug and is not related to the investigational product or procedure, possible if the adverse event is determined to be potentially related to the investigational product or procedure, and an alternative aetiology is equally or less likely compared with potential relationship to investigational product or procedure, or probable if there is a strong relationship to investigational product or procedure or recurs on re-challenge, and another aetiology is unlikely, or highly probably if there is no other reasonable medical explanation for the event.

\section{Statistical considerations}

Descriptive statistics will be reported as mean (SD), median (range) and proportions as appropriate. Data will be analysed as intention-to-treat and hypothesis testing performed with t-test, Wilcoxon rank-sum test, $\chi^{2}$ and Fisher's exact test as appropriate. Pre-planned secondary analyses will include per-protocol analyses, effect modification by timing of colonoscopy (ie, morning vs afternoon procedures), history of constipation or IBS-C and bowel preparation used at index colonoscopy. A sensitivity analysis of the primary outcome using a cut-off value of $\geq 7$ to define adequate bowel preparation will also be performed.

The primary strategy for dealing with missing data will be avoidance through adequate training of research staff in data collection, use of centralised data capture and regular audits of data integrity by the central site. In addition, given the short study duration which ends on completion of the colonoscopy, we do not anticipate issues with dropouts, although this will be taken into account for the sample size calculation. For patients who do not present for colonoscopy, contact will be made by telephone to enquire as to the reason, whether it was related to the study medication, and documented appropriately. In the event the patient did not present for colonoscopy for reasons unrelated to bowel preparation, such as forgetting the appointment, the patient can remain in the study by rebooking the procedure and using the same randomised bowel preparation to limit dropouts.

Sample size was calculated as follows. Assuming 70.0\% adequacy among those randomised to regimen $\mathrm{A}$, $87.5 \%$ adequacy among those randomised to regimen $\mathrm{B}$, significance of 0.05 and a power of $0.80,85$ patients are required in each group (total $=170$ ). An adequacy rate of $70 \%$ was selected based on the existing literature. ${ }^{17} 19$ Additional factors considered in arriving at this figure include the use of low-volume sodium phosphate, ${ }^{17}$ inadequate use of split dosing ${ }^{17}$ and poor intake of PEG-ELS, ${ }^{19}$ all at the index colonoscopy of prior studies. Accordingly, this would indicate that subjects enrolled in the current trial will represent a more difficult to cleanse population, given they would have failed modern-day split-dose bowel preparation despite being compliant to PEG-ELS intake, unlike prior studies. An adequacy rate of $87.5 \%$ was selected based on a $25 \%$ relative increase in adequacy to be considered clinically significant. Based on a target sample size of 170 and a conservative $15 \%$ dropout, 196 subjects will be recruited for the study.

\section{ETHICS AND DISSEMINATION}

There are no specific ethical considerations in the study protocol beyond that inherent in any clinical trial. The study protocol does not target vulnerable populations and the study medication is generally considered to have 
a low risk for adverse events. The protocol has received regulatory approval from Health Canada (Clinical Trial Application \#HC6-24-c200341), Research Ethics Board approval from all participating centres (Western University \#108472, University of Montreal \#Nr CER 17.207, University of Alberta Pro00072349, McGill University \#MP-37-2017-3324), and will be conducted according to Good Clinical Practice guidelines. ${ }^{33}$ Informed consent will be obtained by research personnel prior to enrollment according to the Tri-Council Policy Statement: Ethical Conduct for Research Involving Humans (online supplementary appendix D) ${ }^{34}$ The study is registered with the National Institute of Health's ClinicalTrials.gov.

The final dataset will be hosted at the central site, Western University, in a deidentified form containing only subject ID numbers. Local sites will not transfer the master list linking subject ID numbers with hospital personal identification numbers to the central site to protect subject confidentiality. The findings of the study will be presented at a major international gastroenterology conference, such as the United European Gastroenterology Week or Digestive Disease Week. In addition, the findings of the study will be published in peer reviewed journals for widespread dissemination. Authorship will be granted for individuals who contribute substantially to the study, including study design, protocol refinement, recruitment, data collection, statistical analysis and manuscript preparation. There are no plans to use professional writers.

\section{Patient and public involvement}

Patients and the public were not involved in the development of the protocol. The protocol will assess the burden of the intervention through a validated questionnaire all patients complete that will determine the overall difficulty of taking the bowel preparation. Copies of the manuscript can be made available to patients after publication on request.

\section{Author affiliations \\ ${ }^{1}$ Program for Experimental Medicine, Western University, London, Ontario, Canada ${ }^{2}$ Division of Gastroenterology, London Health Sciences Centre, London, Ontario, Canada \\ ${ }^{3}$ Division of Gastroenterology, Centre Hospitalier de L'Universite de Montreal, Montreal, Quebec, Canada \\ ${ }^{4}$ Division of Gastroenterology, University of Alberta, Edmonton, Alberta, Canada \\ ${ }^{5}$ Division of Gastroenterology, McGill University, Montreal, Quebec, Canada}

Contributors The protocol was written by MSLS with input from AB, RS, DvR and $\mathrm{MM}$. All investigators critically revised iterations of the protocol and approved the final version. The manuscript was written by MSLS and critically revised by AB, RS, DvR, MM and CMD. All authors agree to the final version of the manuscript.

Funding This work was supported by the Academic Medical Organization of Southwestern Ontario (AMOSO), which provided salary support to MSLS, and Pharmascience, which provided PEG-ELS and bisacodyl in kind, and an arms-length unrestricted grant to support study expenses. As the commercial manufacturer of the study medications, Pharmascience provided limited administrative support for the Clinical Trials Application to Health Canada.

Competing interests Pharmascience were not involved in the study protocol and will not have access to study data nor its analysis, interpretation or decision to publish. MSLS has served as a speaker and participated in an advisory board for an unrelated topic for Pharmascience (ie, bile acid diarrhea). DvR has participated in an advisory board and has received research support from Pharmascience.

Patient consent for publication Not required.

Provenance and peer review Not commissioned; externally peer reviewed.

Open access This is an open access article distributed in accordance with the Creative Commons Attribution Non Commercial (CC BY-NC 4.0) license, which permits others to distribute, remix, adapt, build upon this work non-commercially, and license their derivative works on different terms, provided the original work is properly cited, appropriate credit is given, any changes made indicated, and the use is non-commercial. See: http://creativecommons.org/licenses/by-nc/4.0/.

\section{REFERENCES}

1. Saltzman JR, Cash BD, Pasha SF, et al. Bowel preparation before colonoscopy. Gastrointest Endosc 2015;81:781-94.

2. Rex DK. Optimal bowel preparation--a practical guide for clinicians. Nat Rev Gastroenterol Hepatol 2014;11:419-25.

3. Johnson DA, Barkun AN, Cohen LB, et al. Optimizing adequacy of bowel cleansing for colonoscopy: recommendations from the U.S. multi-society task force on colorectal cancer. Gastrointest Endosc 2014;80:543-62.

4. Froehlich F, Wietlisbach V, Gonvers JJ, et al. Impact of colonic cleansing on quality and diagnostic yield of colonoscopy: the European Panel of Appropriateness of Gastrointestinal Endoscopy European multicenter study. Gastrointest Endosc 2005;61:378-84.

5. Clark BT, Rustagi T, Laine L. What level of bowel prep quality requires early repeat colonoscopy: systematic review and meta-analysis of the impact of preparation quality on adenoma detection rate. Am J Gastroenterol 2014;109:1714-23.

6. Sherer EA, Imler TD, Imperiale TF. The effect of colonoscopy preparation quality on adenoma detection rates. Gastrointest Endosc 2012;75:545-53.

7. Harewood GC, Sharma VK, de Garmo P. Impact of colonoscopy preparation quality on detection of suspected colonic neoplasia. Gastrointest Endosc 2003;58:76-9.

8. Lebwohl B, Kastrinos F, Glick M, et al. The impact of suboptimal bowel preparation on adenoma miss rates and the factors associated with early repeat colonoscopy. Gastrointest Endosc 2011;73:1207-14.

9. Chokshi RV, Hovis CE, Hollander T, et al. Prevalence of missed adenomas in patients with inadequate bowel preparation on screening colonoscopy. Gastrointest Endosc 2012;75:1197-203.

10. Hong SN, Sung IK, Kim JH, et al. The Effect of the Bowel Preparation Status on the Risk of Missing Polyp and Adenoma during Screening Colonoscopy: A Tandem Colonoscopic Study. Clin Endosc 2012;45:404-11.

11. Yee R, Manoharan S, Hall C, et al. Optimizing bowel preparation for colonoscopy: what are the predictors of an inadequate preparation? Am J Surg 2015;209:787-92.

12. MacPhail ME, Hardacker KA, Tiwari A, et al. Intraprocedural cleansing work during colonoscopy and achievable rates of adequate preparation in an open-access endoscopy unit. Gastrointest Endosc 2015;81:525-30.

13. Dik VK, Moons LM, Hüyük M, et al. Predicting inadequate bowel preparation for colonoscopy in participants receiving split-dose bowel preparation: development and validation of a prediction score. Gastrointest Endosc 2015;81:665-72.

14. Ben-Horin S, Bar-Meir S, Avidan B. The outcome of a second preparation for colonoscopy after preparation failure in the first procedure. Gastrointest Endosc 2009;69(3 Pt 2):626-30.

15. Ness RM, Manam R, Hoen $\mathrm{H}$, et al. Predictors of inadequate bowel preparation for colonoscopy. Am J Gastroenterol 2001;96:1797-802.

16. Hookey LC, Vanner SJ. Pico-salax plus two-day bisacodyl is superior to pico-salax alone or oral sodium phosphate for colon cleansing before colonoscopy. Am J Gastroenterol 2009;104:703-9.

17. Ibáñez M, Parra-Blanco A, Zaballa P, et al. Usefulness of an intensive bowel cleansing strategy for repeat colonoscopy after preparation failure. Dis Colon Rectum 2011;54:1578-84.

18. Martel M, Barkun AN, Menard C, et al. Split-Dose Preparations Are Superior to Day-Before Bowel Cleansing Regimens: A Meta-analysis. Gastroenterology 2015;149:79-88.

19. Kim JW, Han JH, Boo SJ, et al. Rescue bowel preparation: same day $2 \mathrm{~L}$ polyethylene glycol addition, not superior to bisacodyl addition 7 days later. Dig Dis Sci 2014;59:2215-21.

20. Gimeno-García AZ, Hernandez G, Aldea A, et al. Comparison of Two Intensive Bowel Cleansing Regimens in Patients With Previous 
Poor Bowel Preparation: A Randomized Controlled Study. Am J Gastroenterol 2017;112:951-8.

21. Rex DK, Bond JH, Winawer S, et al. Quality in the technical performance of colonoscopy and the continuous quality improvement process for colonoscopy: recommendations of the U.S. Multi-Society Task Force on Colorectal Cancer. Am J Gastroenterol 2002;97:1296-308.

22. Parmar R, Martel M, Rostom A, et al. Validated Scales for Colon Cleansing: A Systematic Review. Am J Gastroenterol 2016;111:197-204.

23. Rex DK, Schoenfeld PS, Cohen J, et al. Quality indicators for colonoscopy. Am J Gastroenterol 2015;110:72-90

24. Cohen J, Pike IM. Defining and measuring quality in endoscopy. Gastrointest Endosc 2015;81:1-2.

25. Calderwood AH, Jacobson BC. Comprehensive validation of the Boston Bowel Preparation Scale. Gastrointest Endosc 2010;72:686-92.

26. Calderwood AH, Schroy PC. 3rd, Lieberman DA, Logan JR, Zurfluh M, Jacobson BC. Boston Bowel Preparation Scale scores provide a standardized definition of adequate for describing bowel cleanliness. Gastrointestinal endoscopy 2014;80:269-76.

27. Charlson ME, Pompei P, Ales KL, et al. A new method of classifying prognostic comorbidity in longitudinal studies: development and validation. J Chronic Dis 1987;40:373-83.
28. Longstreth GF, Thompson WG, Chey WD, et alGastroenterology 2006;130:1480-91.

29. Lai EJ, Calderwood AH, Doros G, et al. The Boston bowel preparation scale: a valid and reliable instrument for colonoscopyoriented research. Gastrointest Endosc 2009;69(3 Pt 2):620-5.

30. Lawrance IC, Willert RP, Murray K. A validated bowel-preparation tolerability questionnaire and assessment of three commonly used bowel-cleansing agents. Dig Dis Sci 2013;58:926-35.

31. Pasha SF, Shergill A, Acosta RD, et al. The role of endoscopy in the patient with lower GI bleeding. Gastrointest Endosc 2014;79:875-85.

32. Rahimi RS, Singal AG, Cuthbert JA, et al. Lactulose vs polyethylene glycol 3350--electrolyte solution for treatment of overt hepatic encephalopathy: the HELP randomized clinical trial. JAMA Intern Med 2014;174:1727-33.

33. International Council for Harmonisation of Technical Requirements for Pharmaceuticals for Human Use (ICH), ICH Harmonised Guideline, Integrated Addendum to ICH E6(R1): Guideline for Good Clinical Practice, E6(R2). 2016.

34. Canadian Institutes of Health Research NSaECoC, and Social Sciences and Humanities Research Council of Canada. Ethical Conduct for Research Involving Humans: Tri-Council Policy Statement, 2010. 